\title{
Solutes as controllers of endomembrane dynamics
}

Amra Saric (10 ${ }^{\prime}$ and Spencer A. Freeman $(10)^{2,3 凶}$

Membrane dynamics, such as those associated with intracellular traffic, require controlled deformation of the membrane. There is now evidence that the regulation of the luminal fluid composition - via solute fluxes — prominently impacts dynamic properties of endomembranes by modulating their tension. This has important consequences for the function of the endo-lysosomal system.

C6 regulated solute transport across endomembranes affords cells the means to initiate or override membrane remodelling events 2
${ }^{1}$ Neurosciences and Cellular and Structural Biology Division, Eunice Kennedy Shriver National Institute of Child Health and Human Development, National Institutes of Health, Bethesda, MD, USA.

2Program in Cell Biology, Peter Gilgan Centre for Research and Learning, Hospital for Sick Children, Toronto, ON, Canada.

${ }^{3}$ Department of Biochemistry, University of Toronto, Toronto, ON, Canada.

凶e-mail:spencer.freeman@ sickkids.ca

https://doi.org/10.1038/ s41580-021-00334-0

\section{Solute transport and membrane tension}

Generally, mammalian membranes are not capable of stretching beyond $3-5 \%$, and therefore their tension needs to be well regulated. That cells are capable of counteracting sudden changes in tension is best appreciated at the plasma membrane (PM). High tension is relieved by expanding the membrane surface area by unfurling membrane undulations such as caveolae and microvilli, or by delivering membrane reservoirs from endosomes/ lysosomes that can mobilize to fuse with the strained PM. Conversely, sudden decreases in PM tension result in increased bulk-endocytic uptake, a means to remove slack membrane and reduce surface area. For some cells, maintaining this balance is an ongoing normal part of life. For example, synaptic vesicle fusion triggered by action potentials in neurons is coupled to compensatory rapid endocytosis ${ }^{1}$.

While regulation of PM tension is well studied, little is known about tension experienced by membrane-bound organelles. However, membrane tension in endomembranes, and in the endo-lysosomal system in particular, is expected to be functionally important, as these organelles are highly dynamic, undergoing frequent fusion, fission and often extreme membrane remodelling.

During endocytosis, cargo receptors and their ligands are taken up into endosomes along with fluid, and promptly sorted for recycling or lysosomal degradation. These events are associated with major remodelling of the endomembrane by curvature-sensing protein coats and membrane-deforming polymers, resulting in the formation of structures with high membrane curvature: tubulo-vesicular carriers and intralumenal vesicles (ILVs), respectively. The tubulation and vesiculation events only proceed if the endomembrane tension is permissive of the required deformation; high tension prevents membranes from deforming ${ }^{2,3}$ while reducing tension initiates membrane remodelling ${ }^{4}$.

A wealth of studies on cell volume regulation revealed that PM tension is exquisitely controlled by solute transport pathways. Because the PM is permeable to water, solute transport is a rapid method to direct water flow through osmosis and consequently alter the hydrostatic pressure on the lipid bilayer. This, in turn, is proportional to the amount of lateral tension that a membrane experiences. Endo-lysosomal organelles are also water permeable and replete with unique solute transporters (that is, solute carriers), channels and pumps, which can regulate hydrostatic pressure in these organelles; some of these solute-transporting proteins are regulated by lipids that sense osmotic and metabolic changes in the cell. Thus, regulated solute transport across endomembranes affords cells the means to initiate or override membrane remodelling events. In the following sections we discuss the emerging functional importance of these mechanisms in the endo-lysosomal system, highlighting their reported roles in endocytic sorting, lysosome homeostasis and controlling infection.

\section{Endocytic recycling}

Early endocytic organelles - including endosomes, macropinosomes and phagosomes - contain extracellular fluid, the major osmolytes of which are $\mathrm{Na}^{+}$and $\mathrm{Cl}^{-}$. These monovalent ions are much more abundant in the extracellular fluid than in the cytosol and undergo efflux from the early endocytic organelles into the cytosol, down their electrochemical gradient. Yet, very little is known about their transport across endomembranes. $\mathrm{Na}^{+}$exits early endosomes via two-pore channels (TPCs) and possibly by other means (for example TRPML channels). This process is accompanied by $\mathrm{Cl}^{-}$efflux in order to maintain electroneutrality. Importantly, the efflux of $\mathrm{Na}^{+}$and $\mathrm{Cl}^{-}$from endosomes also drives the loss of water from these compartments, effectively lowering hydrostatic pressure and membrane tension.

The efflux of $\mathrm{Na}^{+}$from endosomes is controlled by a rare lipid species, phosphatidylinositol 3,5-bisphosphate $\left(\operatorname{PtdIns}(3,5) \mathrm{P}_{2}\right)$, that activates cation channels including TPCs and TRPMLs. PtdIns $(3,5) \mathrm{P}_{2}$ is generated on the cytoplasmic leaflet of endosomes by the lipid kinase PIKfyve. As such, PIKfyve inhibitors block $\mathrm{Na}^{+}$efflux 
from endo-lysosomes and cause swelling of these organelles, reflecting an inability to expel luminal water. Under such circumstances, membrane loss due to initial sorting events quickly increases the hydrostatic pressure and hence membrane tension of the endosome, hindering further recruitment of membrane remodelling complexes. In principle, the tensed organelles may undergo fusion to modestly increase the surface-to-volume ratio and thereby relieve some of the tension, but this ultimately does not correct the underlying problem, as solutes remain trapped in the lumen. As a result, cells that cannot drive efflux of water from endocytic compartments show impaired receptor recycling and degradative sorting and consequently become unresponsive to extracellular cues ${ }^{4}$.

\section{Lysosome homeostasis}

By the time endocytic fluid reaches lysosomes, it is substantially distilled and becomes highly acidic, owing to the constitutive $\mathrm{H}^{+}$-pumping activity of vacuolar ATPases. The low luminal $\mathrm{pH}$ is critical for the optimal function of most lysosomal hydrolases, enzymes that break down incoming macromolecules into their constituent building blocks. The generation of such building blocks increases the osmotic activity of the lysosomal lumen. These solutes must therefore be transported out of the lysosomes making them available for subsequent cytoplasmic metabolism and preventing the imposition of an additional osmotic load. Impairment of solute efflux is associated with swelling that arrests membrane remodelling, effectively demonstrated by 'sucrosomes' - lysosomes loaded with sucrose, a non-transportable disaccharide that cannot be broken down by endogenously expressed lysosomal hydrolases. Such lysosomes swell and become devoid of ILVs. Upon the addition of exogenous invertase, an enzyme that hydrolyses sucrose into transportable monosaccharides, lysosomal swelling is resolved by way of tubulation/vesiculation ${ }^{6}$. As a pathophysiological corollary, distended lysosomes manifest in numerous lysosomal storage disorders where solutes fail to be translocated across the lysosomal membrane and thus accumulate within the lysosomal lumen. This suggests that misregulation of solute transport could be one of the mechanisms underlying lysosomal storage disorders.

Accurate measurements of lysosomal ion concentrations, apart from $\mathrm{H}^{+}$, have proven difficult to obtain, but available sources suggest that monovalent ions remain the most abundant osmolytes of lysosomes. This raises the possibility that fluxes of these ions regulate lysosomal tubulation, cargo traffic and fission in a similar fashion as they do for endosomes. Indeed, this was demonstrated for autolysosomes - structures formed through fusion of lysosomes with autophagosomes during autophagy. In this context, when autolysosomes undergo fission to regenerate lysosomes, they do so via extensive tubulation ${ }^{7}$. This process should only be initiated after the complete digestion of autophagic substrates and the efflux of the released metabolites. The regulated fluxes of small organic degradation products and of monovalent ions could provide a means for coordinating these events. This regulation could be achieved via the nutrient sensor, mTOR, which is able to sense amino acid presence in the lysosomal lumen and has an under-appreciated role in inhibiting TPCs ${ }^{8}$. Thus, mTOR is exquisitely situated at the juncture of amino acid sensing, $\mathrm{Na}^{+}$efflux and lysosome tubulation. It is tempting to speculate that mTOR coordinates the timing of autolysosome fission by sensing the completion of autophagic degradation, to activate TPCs thereby enabling membrane remodelling for lysosome re-establishment.

\section{Infection and endomembrane tension}

The exploitation of endomembrane tension via solute flux has merit as a therapeutic strategy during infection. Enveloped viruses are taken up into endosomes and deliver their genome into the host cell cytosol by inserting fusogenic peptides into the endomembrane to drive fusion with the viral envelope. This fusion event is associated with membrane deformation events, which, as discussed above, are opposed by high endomembrane tension. Indeed, inhibiting PIKfyve or TPCs blocks the entry of SARS-CoV-2 and Ebola virus.

Conversely, some bacteria like Helicobacter pylori and Listeria monocytogenes can establish pathogensupporting niches in cytoplasmic vacuoles of host cells. These highly spherical, enlarged vesicles are likely under high membrane tension that opposes their remodelling and resolution by host trafficking complexes. From the pathogen's perspective, this would ensure that its niche remains protected, allowing the microorganism to thrive.

\section{Conclusion}

All forms of endocytosis involve pinocytosis, the uptake of fluid. Despite ongoing fluid uptake, the volume of the endocytic pathway and that of the cell remain unchanged $^{9}$. We have highlighted the emerging view that cells handle endocytic fluid through coordinated solute transport, accompanied by osmotic shifts that direct water flow, to regulate endomembrane tension and trafficking. In the future it will be interesting to address how these processes are dysregulated in diseases, including lysosomal storage disorders or during pathogenic infection.

1. Watanabe, S. et al. Ultrafast endocytosis at mouse hippocampal synapses. Nature 504, 242-247 (2013).

2. Simunovic, M. \& Voth, G. A. Membrane tension controls the assembly of curvature-generating proteins. Nat. Commun. 6, 7219 (2015).

3. Mercier, V. et al. Endosomal membrane tension regulates ESCRT-IIIdependent intra-lumenal vesicle formation. Nat. Cell. Biol. 22 947-959 (2020).

4. Freeman, S. A. et al. Lipid-gated monovalent ion fluxes regulate endocytic traffic and support immune surveillance. Science 367 , 301-305 (2020).

5. Wang, X et al. TPC proteins are phosphoinositide- activated sodium-selective ion channels in endosomes and lysosomes. Cell 151, 372-383 (2012).

6. Bright, N. A., Davis, L. J. \& Luzio, J. P. Endolysosomes are the principal intracellular sites of acid hydrolase activity. Curr. Biol. 26, 2233-2245 (2016)

7. Yu, L. et al. Termination of autophagy and reformation of lysosomes regulated by mTOR. Nature 465, 942-946 (2010).

8. Cang, C. et al. mTOR regulates lysosomal ATP-sensitive two-pore $\mathrm{Na}(+)$ channels to adapt to metabolic state. Cell 152, 778-790 (2013).

9. Steinman, R. M., Brodie, S. E. \& Cohn, Z. A. Membrane flow during pinocytosis. A stereologic analysis. J. Cell. Biol. 68, 665-687 (1976).

Acknowledgements

We thank Dr. Sergio Grinstein for valuable editing of this work. S.A.F. is supported by a CIHR Project Grant PJT-169180.

\section{Competing interests}

The authors declare no competing interests. 